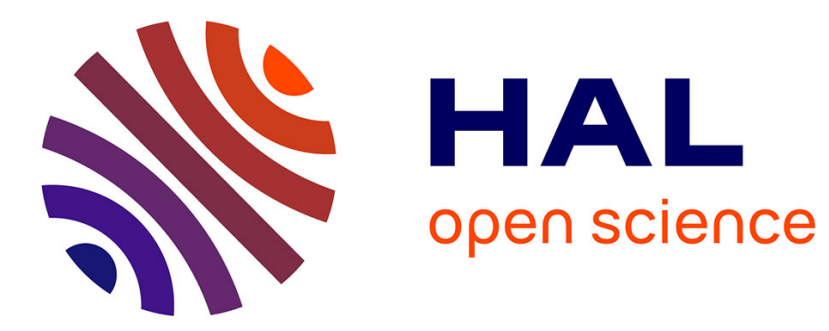

\title{
Multi-system Nernst-Michaelis-Menten model applied to bioanodes formed from sewage sludge
}

Mickaël Rimboud, Elie Desmond-Le Quemener, Benjamin Erable, Théodore Bouchez, Alain Bergel

\section{- To cite this version:}

Mickaël Rimboud, Elie Desmond-Le Quemener, Benjamin Erable, Théodore Bouchez, Alain Bergel. Multi-system Nernst-Michaelis-Menten model applied to bioanodes formed from sewage sludge. Bioresource Technology, 2015, Microbial Fuel Cells, 195, pp.162-169. 10.1016/j.biortech.2015.05.069 hal01324124

\section{HAL Id: hal-01324124 \\ https://hal.science/hal-01324124}

Submitted on 28 Jun 2016

HAL is a multi-disciplinary open access archive for the deposit and dissemination of scientific research documents, whether they are published or not. The documents may come from teaching and research institutions in France or abroad, or from public or private research centers.
L'archive ouverte pluridisciplinaire HAL, est destinée au dépôt et à la diffusion de documents scientifiques de niveau recherche, publiés ou non, émanant des établissements d'enseignement et de recherche français ou étrangers, des laboratoires publics ou privés. 


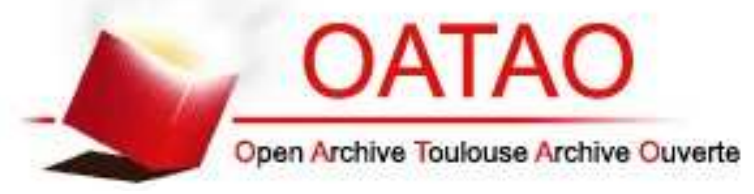

\section{Open Archive TOULOUSE Archive Ouverte (OATAO)}

OATAO is an open access repository that collects the work of Toulouse researchers and makes it freely available over the web where possible.

This is an author-deposited version published in : http://oatao.univ-toulouse.fr/ Eprints ID : 15708

To link to this article : DOI : 10.1016/j.biortech.2015.05.069

URL : http://dx.doi.org/10.1016/j.biortech.2015.05.069

To cite this version : Rimboud, Mickaël and Desmond-Le

Quemener, Elie and Erable, Benjamin and Bouchez, Théodore and

Bergel, Alain Multi-system Nernst-Michaelis-Menten model

applied to bioanodes formed from sewage sludge. (2015)

Bioresource Technology, vol. 195. pp. 162-169. ISSN 0960-8524

Any correspondence concerning this service should be sent to the repository administrator: staff-oatao@ listes-diff.inp-toulouse.fr 


\title{
Multi-system Nernst-Michaelis-Menten model applied to bioanodes formed from sewage sludge
}

\author{
Mickaël Rimboud $^{\mathrm{a}, *}$, Elie Desmond-Le Quemener ${ }^{\mathrm{b}}$, Benjamin Erable ${ }^{\mathrm{a}}$, Théodore Bouchez ${ }^{\mathrm{b}}$, Alain Bergel ${ }^{\mathrm{a}}$ \\ a Laboratoire de Génie Chimique, CNRS - Université de Toulouse, 4 allée Emile Monso, 31432 Toulouse, France \\ ${ }^{\mathrm{b}}$ IRSTEA-Unité de Recherche Hydrosystèmes et Bioprocédés, 1 rue Pierre-Gilles de Gennes, CS 10030, 92761 Antony, France
}

\section{H I G H L I G H T S}

- Efficient bioanodes were designed from hydrolyzed sewage sludge.

- Bioanodes electrochemical kinetics were studied using voltammetric analyses.

- Electrochemical systems identified displayed reversible Nernstian kinetics.

- Microbial communities were analysed by 16S-RNA pyrosequencing.

Keywords:

Bioanode

Sewage sludge

Electrochemical kinetics

Microbial fuel cell

Pyrosequencing

\section{A B S T R A C T}

Bioanodes were formed under constant polarization at $-0.2 \mathrm{~V} / \mathrm{SCE}$ from fermented sewage sludge. Current densities reached were $9.3 \pm 1.2 \mathrm{~A} \mathrm{~m}^{-2}$ with the whole fermented sludge and $6.2 \pm 0.9 \mathrm{~A} \mathrm{~m}^{-2}$ with the fermented sludge supernatant. The bioanode kinetics was analysed by differentiating among the contributions of the three redox systems identified by voltammetry. Each system ensured reversible Nernstian electron transfer but around a different central potential. The global overpotential required to reach the maximum current plateau was not imposed by slow electron transfer rates but was due to the potential range covered by the different redox systems. The microbial communities of the three bioanodes were analysed by 16S rRNA gene pyrosequencing. They showed a significant microbial diversity around a core of Desulfuromonadales, the proportion of which was correlated with the electrochemical performance of the bioanodes.

\section{Introduction}

Microbial electrochemical technologies (METs) are emergent environmentally friendly technologies that rely on electron transfer reactions catalysed by the microbial biofilms that spontaneously develop on the electrode surface. Microbial bioanodes are thus able to catalyse the oxidation of the organic matter contained in a large variety of natural environments and industrial or domestic wastes (Pant et al., 2010; Wang et al., 2012). Among these wastes, sewage sludge has been identified as a good candidate to develop and feed microbial bioanodes. Sludge is produced in large quantities all over the world as a side-product of domestic or industrial wastewater treatment. Sludge degradation requires further costly processes (Tyagi and Lo, 2013) but it could be

\footnotetext{
* Corresponding author.

E-mail address: mickael.rimboud@univ-perp.fr (M. Rimboud).
}

valorized in METs, such as microbial fuel cells (MFCs), to produce electrical power.

Raw sludge, however, is mainly composed of microorganisms and consequently constitutes highly complex organic matter, which is difficult to exploit by means of MFCs (Abourached et al., 2014). Various preliminary treatments of sludge have been suggested to improve MFC performance: ultrasonication (Jiang et al., 2009; Oh et al., 2014), alkalinization (Xiao et al., 2011; Oh et al., 2014), microwave treatment or ozonation (Mohd Yusoff et al., 2013). Though a slower process, fermentation has been identified as the simplest and easiest pretreatment of sludge before it is fed into an MFC (Abourached et al., 2014; Yang et al., 2013). Fermentation does not require an external supply of energy or addition of chemicals. Moreover, more and more sewage treatment plants are being equipped with digesters, which use the raw sludge to generate methane and produce fermented sludge on site as a side-product. Fermentation results in increased volatile fatty acid (VFA) contents beneficial to the downstream MFC operation (Freguia et al., 2010; Tyagi and Lo, 2013). 
Nevertheless, the power densities and Coulombic efficiencies displayed by MFCs fed with sewage sludge still remain low (Abourached et al., 2014). Two causes can be distinguished. Firstly, the difficulties proper to the sludge itself and, secondly, the problems linked with the MFC design. As mentioned by Abourached et al. (2014), operations with sewage sludge in MFCs are hindered by non-optimized MFC designs and high internal resistance. Generally speaking, the different processes that interact in an MFC (cathode kinetics, ion migration, possible biofouling of the separator and cathode surfaces, etc.) can drastically limit the global MFC performance and lead to an underestimate of the real potential of the bioanode. The accurate characterization of the bioanode kinetics requires strict electroanalytical conditions using a three-electrode electrochemical set-up (Rimboud et al., 2014). The studies devoted to bioanodes formed in raw effluents, i.e. effluents that have not been modified or diluted by addition of buffer, salts or any other chemicals, that have been performed in electroanalytical conditions remain rare (Rimboud et al., 2014) but they have shown the great potential of the technique, for example when applied to raw effluents coming from paper mills (Ketep et al., 2013).

In this framework, the purpose of the present work was to characterize the capability of sewage sludge to form microbial anodes by breaking free of the possible detrimental interactions that occur with complete MFC devices. In this aim, bioanodes were formed under constant polarization in 3-electrode set-ups. Fermented sewage sludge was used as both the inoculum and the feeding medium for bioanode formation and operation. The bioanodes were characterized by chronoamperometry and cyclic voltammetry; their electrochemical behaviour was discussed in the light of the composition of the bacterial communities analysed by $16 \mathrm{~S}$ rRNA gene pyrosequencing. The current densities provided by the bioanodes formed in these conditions revealed higher potential than shown so far for sewage sludge exploited in METs. The work also exposed a major drawback to be overcome for going ahead with future large-scale development.

\section{Methods}

\subsection{Sludge collection and analysis}

Activated sludge was taken from a sewage treatment plant in Evry, France. The initial pH was 6.7 and the sludge contained $41.3 \mathrm{~g} \mathrm{~L}^{-1}$ of solid matter. Sludge was fermented for 7 days under anaerobic conditions in a tank to favour the degradation of organic matter to volatile fatty acids (VFAs) by acidogenesis. Soluble COD increased from 288 to $1212 \mathrm{mg} \mathrm{L}^{-1}$ during this anaerobic step and the resulting fermented sludge had a $\mathrm{pH}$ of 7 . The fermented sludge was divided into different samples which were frozen for storage. Samples were subsequently defrosted for electrochemical experiments and sludge analyses. Depending on the chosen experimental conditions, electrochemical experiments were performed on the whole sludge or on the sludge supernatant only. The sludge supernatant was obtained by 30 min centrifugation at $4000 \mathrm{rpm}$. VFA concentrations were measured in the supernatant using ion chromatography (DIONEX DX 120, column IONPAC ${ }^{\circledR}$ ICE-AS1 $(9 \times 250 \mathrm{~mm}))$. The mobile phases were heptafluorobutyric acid $\left(0.4 \mathrm{mmol} \mathrm{L}^{-1}\right)$ and TBAOH $\left(5 \mathrm{mmol} \mathrm{L}^{-1}\right)$. Acetic, propionic, butyric, lactic, formic and valeric acids were found to be present in quantities ranging from $10 \mathrm{mg} / \mathrm{L}$ to $500 \mathrm{mg} / \mathrm{L}$.

\subsection{Electrochemical set-up}

Bioanodes were formed and operated in 3-electrode set-ups consisting of two $\mathrm{H}$-shaped compartments separated by an anion exchange membrane (Fumasep ${ }^{\circledR}$ FAA-PK, Germany) having a surface area of $7.1 \mathrm{~cm}^{2}$. The working electrode was a $2 \mathrm{~cm}^{2}$ carbon cloth (Paxitech ${ }^{\circledR}$, France) connected by a platinum wire as the current collector, the counter-electrode was a $10 \mathrm{~cm}^{2}$ platinum grid and the reference electrode was a saturated calomel electrode (SCE, potential $0.24 \mathrm{~V}$ vs. SHE). The working and reference electrodes were in the anodic compartment while the counter-electrode was in the cathodic compartment (see scheme in Rimboud et al., 2015). The anodic medium was $500 \mathrm{~mL}$ of fermented sludge of $\mathrm{pH} 8.3$ after defrosting, without any addition of buffer, salts or other compound. The catholyte was a $\mathrm{K}_{2} \mathrm{HPO}_{4} 250 \mathrm{mM}$ solution, with $\mathrm{pH}$ adjusted to 8.3 by addition of $\mathrm{HCl} 37 \%$ in order to have the same $\mathrm{pH}$ in both compartments at the start of experiments. The $\mathrm{pH}$ was regularly measured in each compartment throughout the experiments but not controlled; it remained free to drift over time. Anodic $\mathrm{pH}$ remained restricted to values compatible with biofilm well-being, varying between $7.3 \pm 0.2$ and $8.8 \pm 0.5$ depending on the experimental conditions (medium renewal, acetate pulses). Cathodic $\mathrm{pH}$ showed higher variations, from 8.3 to $10.4 \pm 0.7$. The concentration of acetate was determined by means of an enzymatic measurement kit (K-acetak, Libios, France).

\subsection{Procedure for bioanode formation and operation}

The microbial anodes were formed under constant polarization at $-0.2 \mathrm{~V} / \mathrm{SCE}$ using a multichannel potentiostat (Biologic, France, EC-Lab software). Currents were recorded versus time and current densities $J$ were expressed with respect to the geometric surface area of the anode carbon cloth $\left(2 \mathrm{~cm}^{2}\right)$. At some times, the constant polarization was interrupted and cyclic voltammetry was recorded, starting from the polarization potential and scanning to $-0.6 \mathrm{~V}$ and then back to $+0.3 \mathrm{~V} / \mathrm{SCE}$ at $1 \mathrm{mV} \mathrm{s}^{-1}$. Two voltammograms were recorded in succession each time. They were mostly identical and only the second one is reported here for the sake of simplicity.

The bioanodes were first formed in a first batch (cycle 1) using $500 \mathrm{~mL}$ of whole fermented sludge in the anodic compartment. When the current fell down to zero, the bioanodes were removed and placed in a new reactor containing fresh sludge supernatant. Two successive batches were run in sludge supernatant (cycles 2 and 3 ). Each batch was considered ended when the current fell down to zero. Finally, three more cycles (cycles 4-6) were performed with pulses of $15 \mathrm{mM}$ acetate $(2 \mathrm{~mL}$ of acetate $4 \mathrm{M}$ ). No medium change was made during these last three cycles operated with acetate. The acetate concentration of $15 \mathrm{mM}$ was chosen so as to have the same quantity of extractable electrons as with the sludge supernatant. All the electrochemical experiments were conducted in a stove thermostated at $40^{\circ} \mathrm{C}$.

The quantity of electrons extractable per litre from each VFA ( $[e]$, mol $\mathrm{e}^{-} \mathrm{L}^{-1}$ ) was calculated as the product of the VFA concentration by the number of electrons $\left(n_{e}\right)$ produced by the complete oxidation of the VFA to $\mathrm{CO}_{2}$ and protons. The quantities derived for each acid were added to estimate the quantity of electrons extractable per litre of medium.

Coulombic efficiency is defined as the ratio of the quantity of electrons effectively extracted to the quantity that could theoretically be released by complete oxidation of the substrate. For each cycle of substrate consumption, the quantity of electron extracted was determined by integrating the current with respect to time. The theoretical extractable quantity was calculated from the soluble COD of the fermented sludge (on average $1212 \mathrm{mg} \mathrm{L}^{-1}$, $74 \mathrm{mmol}$ of electrons) for the first three cycles, and from the acetate concentration ( $15 \mathrm{mM}, 60 \mathrm{mmol}$ of electrons) for the last three cycles. 


\subsection{Population analysis}

The bioanodes were collected at the end of the experiments and kept à $-20^{\circ} \mathrm{C}$ until DNA extraction. Total DNA was extracted with the MOBIO PowerSoil ${ }^{\circledR}$ DNA Isolation reagent kit according to the manufacturer's recommendations. Extracted DNA was quantified by fluorometry (QuBit ${ }^{\mathrm{TM}}$ fluorometer with Quant-it ${ }^{\mathrm{TM}}$ assay kit, Invitrogen) and DNA quality was checked by PCR. 16-S amplicon pyrosequencing was performed using a Roche 454 FLX instrument with titanium reagents from the Research and Testing Laboratory (Lubbock, TX) according to RTL protocols (www.researchandtesting.com) with bacterial primer sets 28F (5'-TTTGATCNTGGCTCA G-3')/519R (5'-GTNTTACNGCGGCKGCTG-3').

Subsequent data analyses were conducted following exactly the procedure detailed previously (Rimboud et al., 2015). After filtering, the remaining sequences were used for picking Operational Taxonomic Units (OTUs) using 97\% sequence similarity as the threshold. Sequences identified as Archaea and Eukarya were then discarded as they resulted from unwanted amplification. Finally, the abundance and relative abundance were computed for each OTU and rarefaction curves were generated.

\section{Results and discussion}

\subsection{Sludge composition}

The concentrations of several VFAs (formic, acetic, propionic, butyric and valeric acids) were measured in the supernatant of the fermented sludge, together with lactic acid as another possible fermentation product (Table 1).

No formic or valeric acid was detected. The absence of valeric acid (C5) suggested that the degradation process was well-advanced. The soluble COD evolved from 288 in the raw sludge supernatant to $1212 \mathrm{mg} \mathrm{L}^{-1}$ after fermentation. The final TOC was $426 \mathrm{mg} \mathrm{L}^{-1}$. The four VFAs identified made up respectively $78.0 \%$ and $76.7 \%$ of the soluble COD and TOC. On the basis of the soluble COD, around $74 \mathrm{mmol}$ of electrons could ideally be expected to be extracted from $0.5 \mathrm{~L}$ of sludge supernatant. Among them, $56 \mathrm{mmol}$ of electrons could be extracted from the four VFAs identified (Table 1).

\subsection{Chronoamperometries}

Three bioanodes were formed under constant polarization at $-0.2 \mathrm{~V} / \mathrm{SCE}$ in parallel, in identical conditions. The applied potential of $0.2 \mathrm{~V} / \mathrm{SCE}$ was in the range of the most used values to form bioanodes (Rimboud et al., 2014). The first batch cycle used the whole sludge in the anodic compartment, the second and third cycles were performed with the sludge supernatant. The last three cycles were achieved by adding $15 \mathrm{mM}$ sodium acetate without changing the medium. In each case, a cycle was considered to have ended when the current dropped quickly towards zero (Fig. 1). The cycles performed with sludge renewal lasted for 23-25 days, while acetate pulses lasted for 16-17 days.

Table 1

Proportion of each VFAs in the hydrolyzed biological sludge supernatant.

\begin{tabular}{llllll}
\hline Acid & Formula & $\begin{array}{l}\text { Concentration } \\
\left(\mathrm{mg} \mathrm{L}^{-1}\right)\end{array}$ & $\begin{array}{l}\text { Concentration } \\
(\mathrm{mM})\end{array}$ & $n_{e}$ & {$[e](\mathrm{mM})$} \\
\hline Formic & $\mathrm{CHO}_{2}$ & nd & nd & 2 & 0 \\
Acetic & $\mathrm{C}_{2} \mathrm{H}_{4} \mathrm{O}_{2}$ & 599.2 & 10.0 & 8 & 79.8 \\
Propionic & $\mathrm{C}_{3} \mathrm{H}_{6} \mathrm{O}_{2}$ & 157.8 & 2.1 & 14 & 29.8 \\
Butyric & $\mathrm{C}_{4} \mathrm{H}_{8} \mathrm{O}_{2}$ & 17.9 & 0.2 & 20 & 4.0 \\
Lactic & $\mathrm{C}_{3} \mathrm{H}_{6} \mathrm{O}_{3}$ & 17.5 & 0.2 & 12 & 2.3 \\
Valeric & $\mathrm{C}_{5} \mathrm{H}_{10} \mathrm{O}_{2}$ & nd & nd & 26 & 0 \\
\hline
\end{tabular}
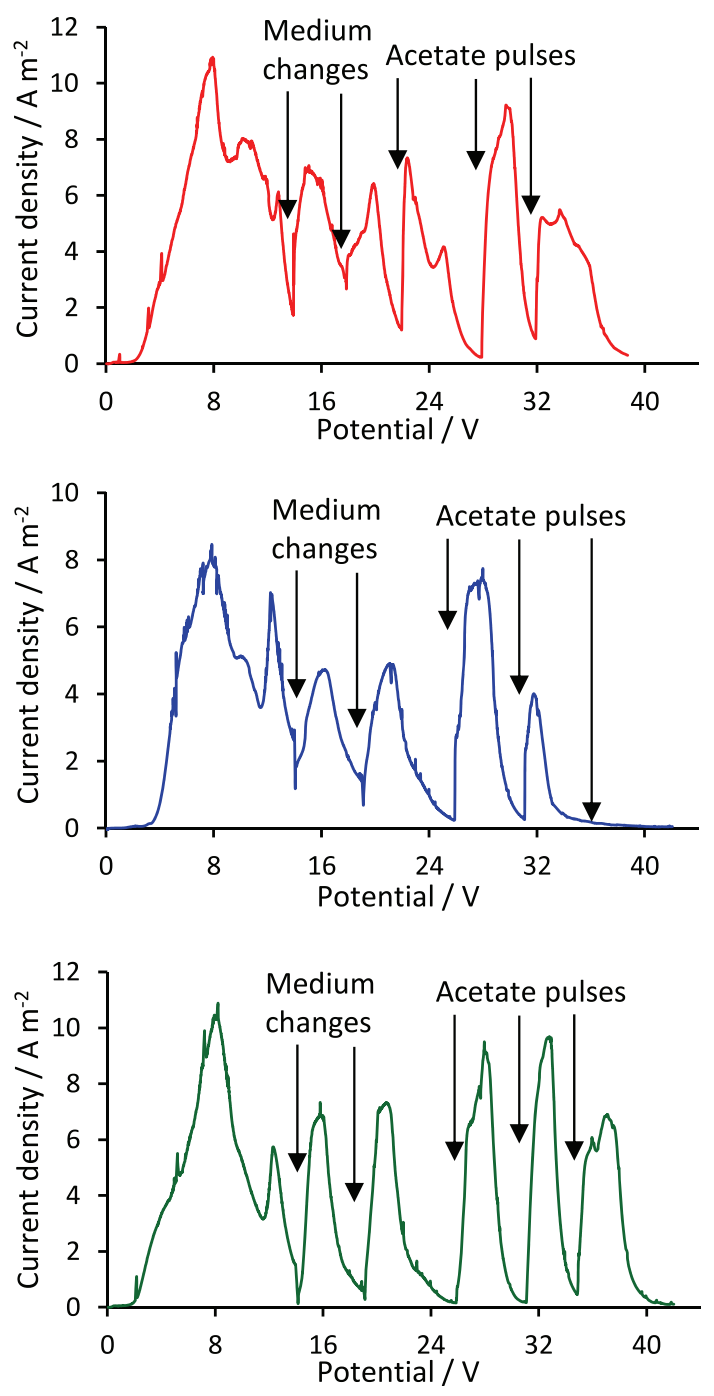

Fig. 1. Chronoamperometries under constant polarization at $-0.2 \mathrm{~V} / \mathrm{SCE}$. The cycles were considered to have ended when the current dropped quickly towards zero. The duration of each cycle could consequently differ slightly from one electrode to another.

The $\mathrm{pH}$ in the anodic compartment underwent fast neutralization after medium change, decreasing from 8.3 to $7.3 \pm 0.2$ in around $24 \mathrm{~h}$. In parallel, a slight alkalinization occurred in the cathodic compartment as the $\mathrm{pH}$ increased from 8.3 to $8.8 \pm 0.1$. In contrast, during the last three cycles performed with acetate pulses and no medium renewal, a progressive alkalinization was observed in both anodic and cathodic compartments. The $\mathrm{pH}$ reached $8.8 \pm 0.5$ and $10.4 \pm 0.7$ in the anodic and cathodic compartments respectively. A similar alkalinization of the anodic medium fed by acetate pulses has previously been observed with biofilms grown in compost leachate or paper mill effluents (Cercado et al., 2013; Ketep et al., 2013). It has been attributed to spontaneous reactions induced by the addition of acetate in non-buffered complex media, because the alkalinization occurred in the same manner in the absence of electrochemical phenomena. As previously observed, this alkalinization did not inhibit bioanode operation (Pocaznoi et al., 2012b).

The three electrodes displayed very similar electrochemical performance. They produced higher current density and higher Coulombic efficiency during the first cycle than in the second and third cycles (Fig. 2). The average values were $9.3 \pm 1.2 \mathrm{~A} \mathrm{~m}^{-2}$ and $14.4 \pm 2.1 \%$, respectively for the first cycle, while only 

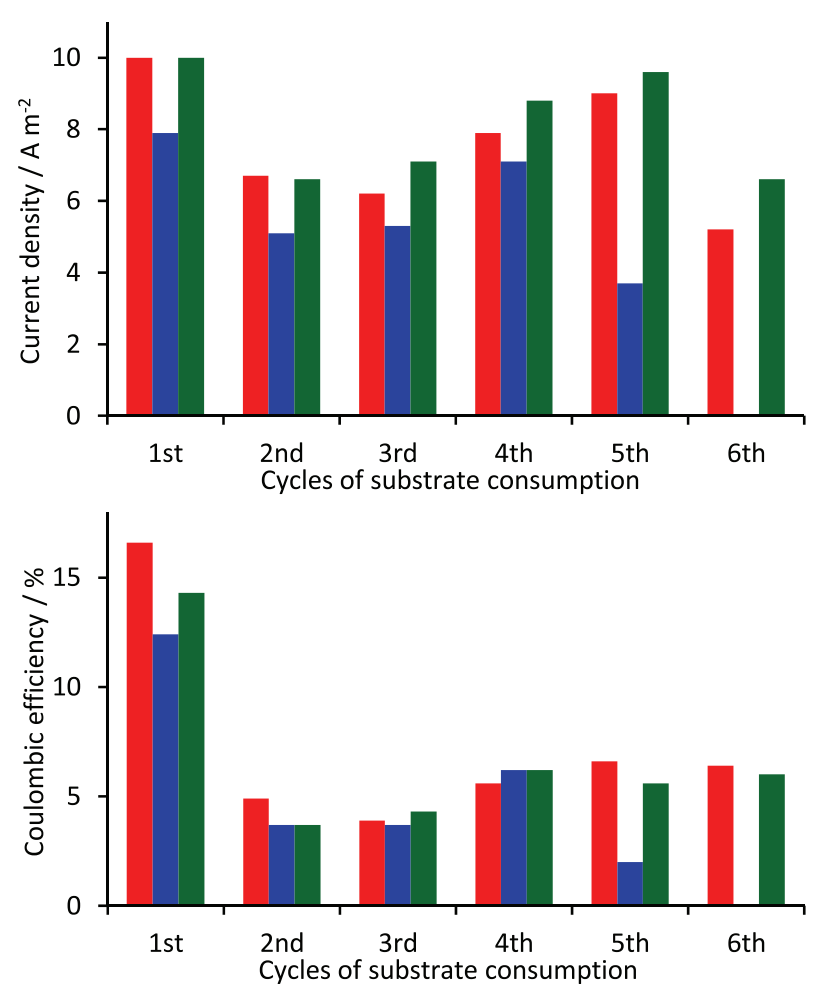

Fig. 2. Maximum current densities $\left(\mathrm{A} \mathrm{m}^{-2}\right)$ and Coulombic efficiencies (\%) per batch cycle displayed by bioanodes 1 (red), 2 (blue) and 3 (green). The first cycles were performed with the whole fermented sludge, the 2nd and 3rd cycles with the fermented sludge supernatant and the last three cycles with pulses of $15 \mathrm{mM}$ sodium acetate. (For interpretation of the references to colour in this figure legend, the reader is referred to the web version of this article.)

$6.2 \pm 0.9 \mathrm{~A} \mathrm{~m}^{-2}$ and $4.0 \pm 0.5 \%$, were reached in the second and third cycles, which led to similar values. This was unusual, as bioanodes ordinarily demonstrate opposite behaviour: the first cycle generally produces lower performance because of the primary establishment of the electroactive biofilm. Here, the only difference between the first and the following two cycles was the presence of the solid matter contained in the whole sludge used in the first cycle. Even though this solid matter settled to the bottom of the reactor very fast (in a few hours) and was not in contact with the bioanodes, it had a beneficial effect on the bioanode performance. The solid matter may have been a source of substrate released to the solution, or it may have provided the bioanode with appropriate microbial species to sustain the biofilm development. Whatever the hypothesis used to explain its positive impact, the presence of solid matter improved the performance of the three bioanodes designed here.

The fourth cycles performed with acetate pulses gave average current density and Coulombic efficiency of $7.9 \pm 0.8 \mathrm{~A} \mathrm{~m}^{-2}$ and $6.0 \pm 0.3 \%$, respectively. Acetate, which cannot be fermented, is known to be an excellent substrate allowing a high electron flux to the anode (Abourached et al., 2014; Chae et al., 2009; Freguia et al., 2010). It was introduced here at the concentration of $15 \mathrm{mM}$ to obtain the same amount of extractable electrons as with the four identified VFAs contained in the sludge supernatant. The increase of the current density observed between cycles 3 and 4 , the concomitant increase in $\mathrm{CE}$ and the shorter cycle time confirmed that acetate was more suitable for electricity production than the longer carbon-chained VFAs contained in the fermented sludge (Chae et al., 2009; Freguia et al., 2010).

However, performance was progressively altered and current densities decreased from $7.9 \pm 0.8$ (first acetate pulse) to $5.9 \pm 0.7 \mathrm{~A} \mathrm{~m}^{-2}$ for two reactors after the third acetate pulse (last cycle), while one reactor provided no current at all. This decrease of the peak currents is inherent in batch conditions and is related to the progressive exhaustion of the medium. Nevertheless, the decrease was fast here, starting as early as the second acetate pulse. In similar experimental conditions but with a different raw medium (leachate formed from a garden compost) stable current peaks have been reported for several acetate pulses (Pocaznoi et al., 2012a). The supernatant of fermented sludge proved to be subject to fast exhaustion here, in a compound that is required to support biofilm activity.

Coulombic efficiencies recorded here (Fig. 2B) were lower than those reported in previous studies dealing with raw sewage sludge (CE $33.3 \pm 1.5 \%$, Abourached et al., 2014). This was a logical result considering the choice of electroanalytical conditions. CE is proportional to the ratio of the electrode surface area to the solution volume. Here, a very small electrode surface area $\left(2 \mathrm{~cm}^{2}\right)$ was used in a fairly large volume of solution $(0.5 \mathrm{~L})$. Such a small ratio should allow the biofilm to develop in the best possible conditions as recently modelled (Rimboud et al., 2014). Electroanalytical conditions were chosen to assess the best possible bioanode kinetics but this was at the price of low CE values. In the present case, CE was even lower because the richness of the sewage sludge favoured alternative oxidation reactions that did not use the anode as a final electron acceptor.

In contrast, as expected, observed current densities were high compared to those found in the literature. The maximum of $9.3 \pm 1.2 \mathrm{~A} \mathrm{~m}^{-2}$ was 4 times higher than one of the best current densities recently reported with raw sewage sludge by Abourached et al. (2014), who claimed a 275\% increase with respect to previous MFC designs. The present study showed that there is still significant room for improvement. Actually, the maximum current density obtained here with the whole fermented sludge $\left(10.8 \mathrm{~A} \mathrm{~m}^{-2}\right)$ was of the same order of magnitude as those reported in the literature where, using similar inocula (wastewater), bioanodes were formed under constant polarization in ideal synthetic culture media that were based on phosphate buffer, ammonium and potassium chloride, with minerals and vitamins (10.3 $\mathrm{A} \mathrm{m}^{-2}$ for Torres et al., 2009; $13 \mathrm{~A} \mathrm{~m}^{-2}$ for Zhu et al., 2014).

The present work reveals that fermented sewage sludge is an excellent candidate to form efficient bioanodes, that dilution in an optimized synthetic medium is not required, and that whole sludge, including the solid matter, even though it settles quickly, is necessary for the best performance.

\subsection{Characterization of the bioanode kinetics by cyclic voltammetry}

Typical cyclic voltammograms recorded with the bioanodes in catalytic (A) and non-catalytic (B) conditions are presented in Fig. 3, together with the first derivative of the catalytic voltammogram (C).

The catalytic signal (Fig. 3A) was observed from around $-0.5 \mathrm{~V} / \mathrm{SCE}$ and the plateau of maximum catalytic current was reached around $-0.3 \mathrm{~V} / \mathrm{SCE}$. The voltammogram obtained in non-catalytic conditions (condition of substrate depletion, Fig. 3B,) displayed at least two clear redox systems, numbered 1 and 2 , and additional less visible signals may also have been present as three signals were identified by deriving the catalytic CV (Fig. 3C). The derivative of the catalytic CV confirmed the presence of the systems numbered 1 and 2 detected on the non-catalytic CV, centred at -0.465 and $-0.375 \mathrm{~V} / \mathrm{SCE}$, respectively. It also revealed a third redox system, 3 , which was partially covered by signal 2 and appeared only as a shoulder.

The Nernst-Michaelis-Menten equation was used to model the catalytic voltammograms. This equation was originally established as the Nernst-Monod equation by Marcus et al. (2007)), but we consider it more accurate to refer to it as the Nernst-Michaelis- 

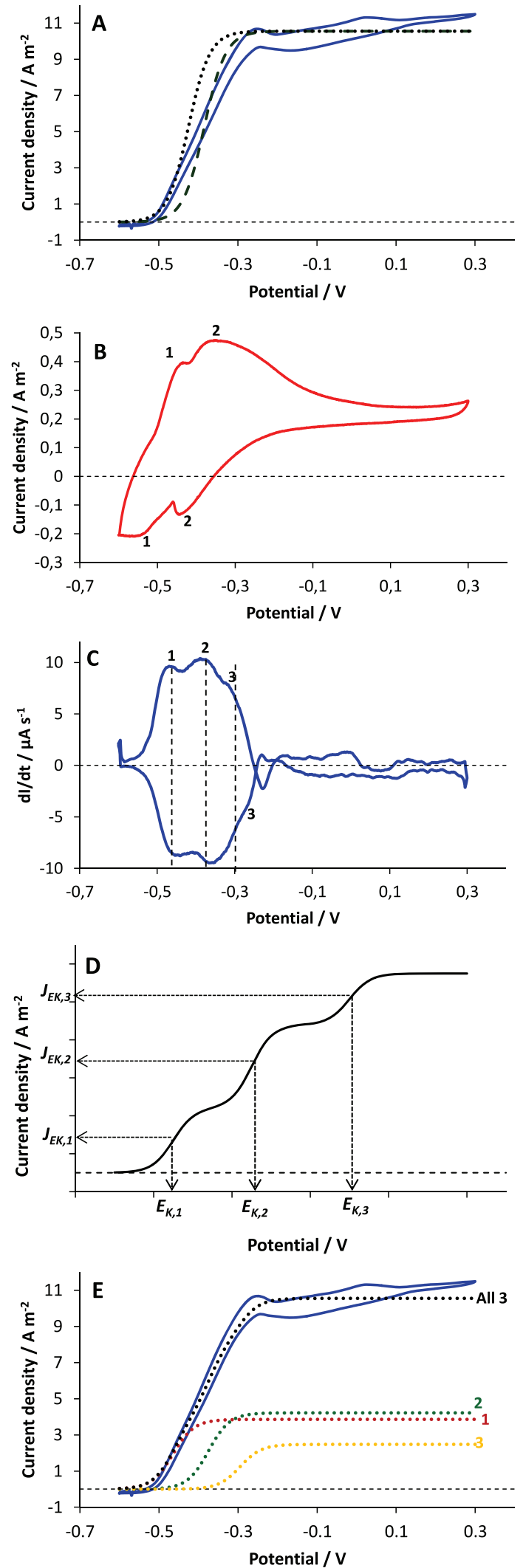

Fig. 3. Cyclic voltammetries recorded with bioanode 3. (A) Catalytic voltammogram recorded during maximum current production after the first medium renewal. (B) Non-catalytic voltammogram recorded after depletion of substrate introduced by the first medium renewal. (C) First derivative analysis of the catalytic voltammogram. (D) Scheme to illustrate the relation between $E_{K, i}$ and $J_{\max , i}$ and their relation to experimental values. (E) Modelling of the catalytic voltammogram from the three signals identified on the first derivative analysis using the Nernst-MichaelisMenten equation.
Menten equation because enzymatic kinetics are involved during the time of voltammetry rather than microbial growth:

$J=J_{\max } \frac{1}{1+\exp \left(-\frac{F}{R T}\left(E-E_{K}\right)\right)}$

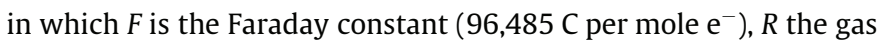
constant $\left(8.3145 \mathrm{~J} \mathrm{~mol}^{-1} \mathrm{~K}^{-1}\right), T$ the temperature $\left(40{ }^{\circ} \mathrm{C}, 313 \mathrm{~K}\right)$ and $E$ the applied potential $(\mathrm{V})$. Two parameters have to be extracted from the experimental catalytic CV: the value of the maximum current density $J_{\max }\left(J_{\max }=10.6 \mathrm{~A} \mathrm{~m}^{-2}\right)$ and value of the potential $E_{K}$ at which $J=J_{\max } / 2\left(E_{K}=-0.385 \mathrm{~V} / \mathrm{SCE}\right)$. The theoretical curve (dashed black line, Fig. $3 \mathrm{~A}$ ) gave a fair representation of the $J-E$ general trend but did not fit it accurately. Curiously, at small overpotentials, the theoretical curve showed slower kinetics than experiment. Such a difference did not make sense physically, because the theoretical curve based on Nernstian reversible electron transfer should lead to the fastest possible electron transfer. To make the representation more theoretically logical, the $E_{K}$ value was artificially adjusted to $-0.425 \mathrm{~V} / \mathrm{SCE} \mathrm{V} / \mathrm{SCE}$ so that the theoretical curve fitted the first part of the experimental one perfectly (dotted black line, Fig. 3A). This second representation was more satisfactory but it differed significantly from the experimental $J-E$ curve at the largest overpotentials.

Here, an original theoretical approach is proposed to improve understanding of the catalytic CV displayed by bioanodes. The CV derivative revealed three distinct redox systems. Logically, a specific Nernst-Michaelis-Menten equation can be attributed to each system with its own $\left(J_{\max , i}, E_{K, i}\right)$ pair of parameters for each. On the CV first derivative (Fig. $3 \mathrm{C}$ ), the potential of each peak corresponds to the point at which the CV derivative stops increasing and starts to decrease, i.e. the inflexion point on the catalytic CV. In Eq. (1), the inflexion point corresponds to the $\left(E_{K}, J_{\max } / 2\right)$ pair (this can be easily demonstrated by calculating the root of the second derivative of Eq. (1)). Consequently the potential of each peak observed on the $\mathrm{CV}$ derivative gave the $E_{K, i}$ value of each system, so $E_{K, 1}=-0.465 \mathrm{~V} / \mathrm{SCE}, E_{K, 2}=-0.375 \mathrm{~V} / \mathrm{SCE}$ and $E_{K, 3}$ was assessed to be $-0.295 \mathrm{~V} / \mathrm{SCE}$. The corresponding $J_{\max , i}$ values were extracted from the experimental catalytic CV as illustrated on the scheme presented in Fig. 3D:

$J_{E_{K}, 1}=J_{\max , 1} / 2$

$J_{E_{K}, 2}=J_{\max , 1}+J_{\max , 2} / 2$

$J_{E_{K}, 3}=J_{\max , 1}+J_{\max , 2}+J_{\max , 3} / 2$

where $J_{E K, 1}, J_{E K, 2}$ and $J_{E K, 3}$ are the current densities corresponding to potentials $E_{K, 1}, E_{K, 2}$, and $E_{K, 3}$, respectively. The three maximum current densities $J_{\max , 1}, J_{\max , 2}$ and $J_{\max , 3}$ were graphically determined as 3.4, 4.4 and $2.7 \mathrm{~A} \mathrm{~m}^{-2}$, respectively.

The three theoretical CVs corresponding to each redox system are plotted in Fig. 3E. The final theoretical CV displayed by the three redox systems is given by summing the three curves. The resulting theoretical curve fitted the whole experimental CV very well, which demonstrated that the experimental signal corresponded to the sum of three Nernstian electron transfers.

This result is of great importance for the basic understanding of biofilm-anode electron transfers. When only one redox system was considered in the model to calculate the theoretical curve, the experimental kinetics appeared less efficient than the assumed Nernstian electron transfer. In contrast, the multi-system model developed here showed that the biofilm-anode electron transfers were fully efficient, as they each different redox systems ensured Nernstian kinetics at the scan rate of $1 \mathrm{mV} \mathrm{s}^{-1}$. Actually, the deviation from a single-system Nernst-Michaelis model was not the mark of less efficient electron transfer, but it was due to the 
involvement of several redox systems, each being centred on a different potential value, and each ensuring reversible electron transfer. In this case, if the objective of further work is to improve the biofilm-anode electron transfer kinetics, the effort should not concern the electrode surface characteristics in priority, because the electrode ensures fast electron transfer with each redox system, but should concentrate on the nature of the redox systems that the biofilm develops.

Much of the literature devoted to microbial bioanodes has shown that several parameters exist to act on the nature of the redox systems expressed by a biofilm. For instance, when bioanodes are formed under chronoamperometry, the applied potential has a direct impact on the number and the potential of the redox systems involved in electron transfer, both in multispecies biofilms (Aelterman et al., 2008; Zhu et al., 2014) and in pure cultures (Zhu et al., 2012). The nature of the substrate has also been shown to affect the enzymatic systems that the cells express to ensure the extracellular electron transfer. For example, different redox systems have been detected by Kaur et al. (2013) when working alternatively with different VFAs as substrates. Acetate and butyrate gave pretty similar CV with a single well-defined redox system, while propionate stimulated several other redox systems.

The multi-system Nernst-Michaelis-Menten theoretical approach developed here should have a considerable impact on the understanding of bioanode kinetics and guide future research endeavours. It would now be of great interest to analyse the bioanode kinetics in view of this new approach that differentiates between the contributions of the various redox systems. The main conclusion drawn here is that each system is very efficient and is able to ensure reversible (Nernstian) electron transfer at a scan rate of $1 \mathrm{mV} \mathrm{s}^{-1}$. The global overpotential that was necessary to reach the maximum current plateau was due to the width of the potential range covered by the different successive systems. In such a case, bioanode improvement should mainly be achieved through control of the biofilm redox composition. Future research endeavours should consequently focus the different operating parameters that can promote the expression of low-potential redox systems. In contrast, since the electron transfer rate of each redox system was fast, working on the electrochemical properties of the electrode surface would be less relevant.

\subsection{Analysis of the microbial communities}

At the end of chronoamperometry, the microbial community of each bioanode was analysed by $16 \mathrm{~S}$ rRNA gene pyrosequencing. The number of operational taxonomic units (OTUs) identified per bioanode was 206, 149 and 108, for bioanodes 1, 2 and 3, respectively. These numbers were used to calculate the different diversity indexes summarized in Table 2.

Bioanode 1 showed the greatest diversity of species represented, with a Chao- 1 index of 238 compared to 200 and 145 for electrodes 2 and 3, respectively. It also presented the most diverse population, as illustrated by a higher Shannon index than the other two electrodes. However, the Simpson indexes calculated for the three anodes were close, ranging between 0.92 (bioanode 2) and 0.94 (bioanode 3 ). This meant that the microbial community of bioanode 1 was the richest, but the evenness of all three biofilms remained similar.

Table 2

Number of species and diversity indexes of the three bioanodes.

\begin{tabular}{lllll}
\hline Bioanode & Number of species & Chao- 1 & Shannon & Simpson \\
\hline 1 & 206 & 238 & 5.46 & 0.94 \\
2 & 149 & 200 & 4.70 & 0.92 \\
3 & 108 & 145 & 4.74 & 0.93 \\
\hline
\end{tabular}

Five phyla dominated the microbial communities. Three phyla: Proteobacteria, Firmicutes and Bacteroidetes, are commonly found among the microbial communities of bioanodes, as pointed out in a recent review and other works (Chae et al., 2009; Zhi et al., 2014). Two other phyla, Chlorobi and Spirochaetes, are not known as common providers of electroactive species.

All the major orders (representing at least $1 \%$ of the sequences on at least one electrode) are presented in the bar charts of Fig. 4. Twenty-three major orders were detected, which accounted for $95 \%$ of identified sequences, but only seven of them accounted for more than $10 \%$ of sequences on at least one electrode: Desulfuromonadales ( $\delta$-proteobacteria), Clostridiales and Thermoanaerobacterales (Firmicutes), Bacteroidales (Bacteroidetes), Ignavibacteriales (Chlorobi), unclassified Spirochaetes and Spirochaetales (Spirochaetes). The table with the abundance of each order is available in the Supplementary material (Fig. S1).

Sequences assigned to Desulfuromonadales were the most numerous in the three bioanodes. This order was the only one represented by more than $10 \%$ of the sequences in each bioanode, ranking respectively second, fourth and first major order in bioanodes 1, 2 and 3. Many bacteria belonging to this order have been identified as efficient electroactive bacteria in the literature, including Geobacter sp. (Bond et al., 2002; Bond and Lovley, 2003; Nercessian et al., 2012), Desulfuromonas sp. (Bond et al., 2002), Geoalkalibacter sp. (Badalamenti et al., 2013; Carmona-Martinez et al., 2013). Phylogenetic identification using the ARB software revealed that the representative sequences of major OTUs belonging to this order were classified as Geobacteraceae related to Geobacter metallireducens (96\% identity for the major OTUs of electrodes 1 and 3), a well-known electroactive bacterium (Bond et al., 2002) and as Desulfuromonadaceae related to Desulfuromonas michiganensis (93\% identity for the third major OTU on electrode 2).

In addition to Desulfuromonadales, some other orders detected here have also been reported as including electroactive species, among them are: Clostridiales (Firmicutes), highly abundant on electrode 1 (19\%) (Niessen et al., 2005), Rhodocyclales (Proteobacteria) abundant on electrodes 2 and 3 (7\% and 9\%) (Lefebvre et al., 2010) and Pseudomonadales abundant on electrode 3 (7\%) (Friman et al., 2012).

Other major orders were unevenly distributed on the different electrodes and included bacteria commonly found in anaerobic sludge, as expected considering the sludge pretreatment (Rivière et al., 2009). Among them, Clostridiales and Thermoanaerobacterales belong to the Firmicutes phylum and Bacteroidales to the Bacteroidetes phylum. Ignavibacterales, which belong to the Chlorobi phylum, were very abundant on electrode 1 . Species belonging to this order have been found previously as the most represented taxa in a bioanode formed with activated sludge (Yoshizawa et al., 2014).

Bioanode 2 revealed drastically lower performance in the fifth cycle and was no longer able to provide current in the last cycle. Two orders of Spirochaetes were found as the second and third major orders in this bioanode, making this phylum the second most represented on this electrode. To the best of our knowledge, Spirochaetes have never been found as a major component of electroactive biofilms. It may consequently be assumed that the presence of Spirochaetes was related to the loss of electroactive performance of the bioanode. The other obvious microbial features that differentiated bioanode 2 from the others was the high abundance of sequences from the order Thermoanaerobacterales (belonging to the Firmicutes phylum). This order, which has never been found as major component of bioanodes, may also be symptomatic of bioanode malfunction. 


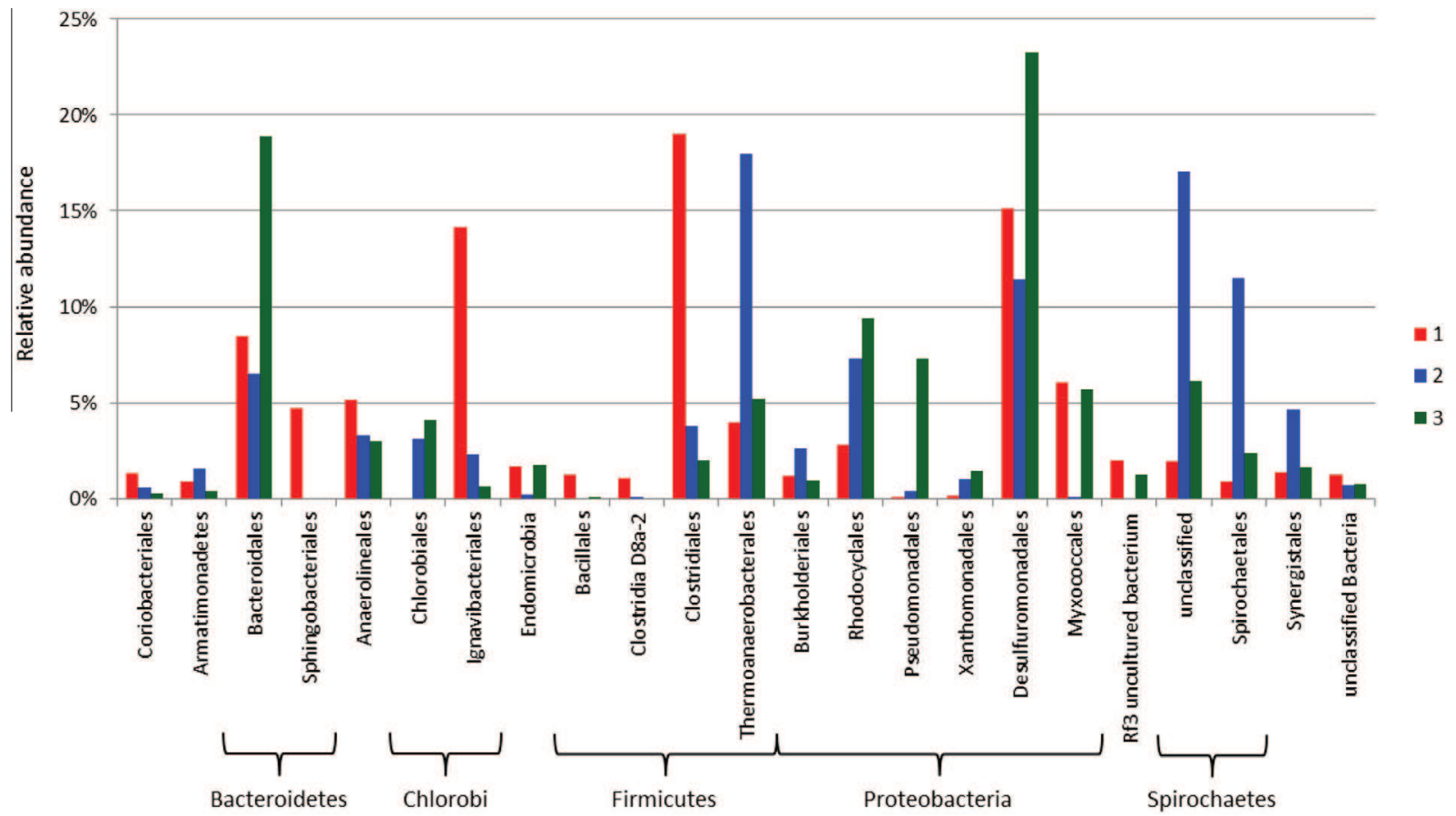

Fig. 4. Relative abundance of bacterial orders found on electrodes 1 (red), 2 (blue) and 3 (green). Only orders represented by at least $1 \%$ sequences in at least one of the samples are shown. They account for $95 \%$ of total identified sequences. Bacterial phyla are indicated when they are represented by several orders. (For interpretation of the references to colour in this figure legend, the reader is referred to the web version of this article.)

In summary, the three bioanodes showed similar statistical biodiversities, as reflected by their diversity indexes (Table 2), but different populations (Fig. 3). However, a correlation was observed between the electrochemical performance and the proportion of Desulfuromonadales. Desulfuromonadales represented $11.4 \%$, $15.1 \%$ and $23.3 \%$ of the total population of the bioanodes 2,1 and 3 , which produced increasing current densities and Coulombic efficiencies (Fig. 2). A similar correlation has recently been reported for bioanodes formed in different media (synthetic culture medium or waste water) and fed with food waste as the substrate (Blanchet et al., 2015). Current densities have proved to be straightforwardly correlated with the enrichment in Geobacteraceae, although the species differed depending on the medium. Here, the electrochemical performance of the bioanodes can be correlated with the proportion of a Desulfuromonadales, while the other phyla, less strongly - or not at all - involved in the electron transfer process, were not present in reproducible ratios in the different bioanodes. Around a core of Desulfuromonadales, which probably provided the bioanodes with the main electroactive species, the microbial community of the other species showed significant dispersion. Finally, the abundance of the phylum Spirochaetes may be related to the loss of electroactivity.

\subsection{Relevance of the three redox systems detected}

As the bioanode microbial communities were dominated by genera related to Geobacteraceae and Desulfuromonadaceae, the voltammetric results were compared to the data available in the literature obtained with bioanodes formed from pure cultures of Geobacter sulfurreducens. The three redox systems found here were centered around $-0.465,-0.375$ and $-0.295 \mathrm{~V} / \mathrm{SCE}$, corresponding to $-0.22,-0.13$ and $-0.05 \mathrm{~V} / \mathrm{SHE}$. In the literature, for G. sulfurreducens bioanodes, Marsili et al. (2010) have detected three redox systems at respectively $-0.235,-0.108,-0.056 \mathrm{~V} / \mathrm{SHE}$ for bioanodes formed under a polarization potential of $0.24 \mathrm{~V} / \mathrm{SHE}$. Zhu et al. (2012), using various polarization potentials, have identified up to seven redox systems; three of them were observed at -0.21 , -0.16 and $-0.09 \mathrm{~V} / \mathrm{SHE}$ with the bioanodes conditioned at -0.25 and $-0.09 \mathrm{~V} / \mathrm{SHE}$. All these systems matched well with the three systems detected here by derivation of the catalytic CV records. Redox systems recorded in other works with G. sulfurreducens bioanodes (Fricke et al., 2008; Katuri et al., 2010) also corresponded with at least two of the values found here. These comparisons indicated that the multi-system model was able to satisfactory fit the whole CV record based on relevant redox potential values. Conversely, CV analysis supported that species related to Desulfuromonadales identified in this work may constitute the main actors of the electrochemical activity of the biofilms.

\section{Conclusions}

Using electroanalytical conditions revealed that fermented raw sewage sludge was an excellent medium to develop efficient bioanodes. The presence of the whole sludge, including solid matter, enhanced the performance, and the reason now needs to be determined by further studies. The innovative kinetic analysis based on a multi-system Nernst-Michaelis-Menten law that was proposed here opened up a new approach for understanding bioanode electron transfers and guiding further practical improvements. Finally, the electrochemical performance was linked to the proportion of Desulfuromonadales in the microbial communities, while the development of Spirochaetes may be related to the loss of electroactivity.

\section{Acknowledgements}

This work benefited from support from the French state, managed by Agence Nationale de la Recherche, within the framework of the French Investissement d'Avenir programme, project BIORARE, number ANR-10-BTBR-02. The authors thank Dr Laure Renvoisé and Suez Environnement for supplying the fermented sludge used in the experiments. 


\section{Appendix A. Supplementary data}

Supplementary data associated with this article can be found, in the online version, at http://dx.doi.org/10.1016/j.biortech.2015.05. 069.

\section{References}

Abourached, C., Lesnik, K.L., Liu, H., 2014. Enhanced power generation and energy conversion of sewage sludge by CEA-microbial fuel cells. Bioresour. Technol. $166,229-234$

Aelterman, P., Freguia, S., Keller, J., Verstraete, W., Rabaey, K., 2008. The anode potential regulates bacterial activity in microbial fuel cells. Appl. Microbiol. Biotechnol. 78, 409-418.

Badalamenti, J.P., Krajmalnik-Brown, R., Torres, C.I., 2013. Generation of high current densities by pure cultures of anode-respiring geoalkalibacter spp. under alkaline and saline conditions in microbial electrochemical cells. mBio 4 e00144-13.

Blanchet, E., Desmond, E., Erable, B., Bridier, A., Bouchez, T., Bergel, A., 2015 Comparison of synthetic medium and wastewater used as dilution medium to design scalable microbial anodes: application to food waste treatment. Bioresour. Technol. 185, 106-115.

Bond, D.R., Holmes, D.E., Tender, L.M., Lovley, D.R., 2002. Electrode-reducing microorganisms that harvest energy from marine sediments. Science 295, 483 485.

Bond, D.R., Lovley, D.R., 2003. Electricity production by Geobacter sulfurreducens attached to electrodes. Appl. Environ. Microbiol. 69, 1548-1555.

Carmona-Martinez, A.A., Pierra, M., Trably, E., Bernet, N., 2013. High current density via direct electron transfer by the halophilic anode respiring bacterium Geoalkalibacter subterraneus. Phys. Chem. Chem. Phys. 15, 19699-19707.

Cercado, B., Byrne, N., Bertrand, M., Pocaznoi, D., Rimboud, M., Achouak, W., Bergel, A., 2013. Garden compost inoculum leads to microbial bioanodes with potential-independent characteristics. Bioresour. Technol. 134, 276-284.

Chae, K.-J., Choi, M.-J., Lee, J.-W., Kim, K.-Y., Kim, I.S., 2009. Effect of different substrates on the performance, bacterial diversity, and bacterial viability in microbial fuel cells. Bioresour. Technol. 100, 3518-3525.

Freguia, S., Teh, E.H., Boon, N., Leung, K.M., Keller, J., Rabaey, K., 2010. Microbial fuel cells operating on mixed fatty acids. Bioresour. Technol. 101, 1233-1238.

Fricke, K., Harnisch, F., Schröder, U., 2008. On the use of cyclic voltammetry for the study of anodic electron transfer in microbial fuel cells. Energy Environ. Sci. 1, $144-147$.

Friman, H., Schechter, A., Nitzan, Y., Cahan, R., 2012. Effect of external voltage on Pseudomonas putida F1 in a bio electrochemical cell using toluene as sole carbon and energy source. Microbiology 158, 414-423.

Jiang, J., Zhao, Q., Zhang, J., Zhang, G., Lee, D.-J., 2009. Electricity generation from bio-treatment of sewage sludge with microbial fuel cell. Bioresour. Technol. $100,5808-5812$.

Katuri, K.P., Kavanagh, P., Rengaraj, S., Leech, D., 2010. Geobacter sulfurreducens biofilms developed under different growth conditions on glassy carbon electrodes: insights using cyclic voltammetry. Chem. Commun. 46, 4758-4760.

Kaur, A., Kim, J.R., Michie, I., Dinsdale, R.M., Guwy, A.J., Premier, G.C., 2013. Microbial fuel cell type biosensor for specific volatile fatty acids using acclimated bacterial communities. Biosens. Bioelectron. 47, 50-55.

Ketep, S.F., Fourest, E., Bergel, A., 2013. Experimental and theoretical characterization of microbial bioanodes formed in pulp and paper mil effluent in electrochemically controlled conditions. Bioresour. Technol. 149, $117-125$.

Lefebvre, O., Ha Nguyen, T.T., Al-Mamun, A., Chang, I.S., Ng, H.Y., 2010. T-RFLP reveals high b-proteobacteria diversity in microbial fuel cells enriched with domestic wastewater. J. Appl. Microbiol. 109, 839-850.
Marcus, A.K., Torres, C.I., Rittman, B.E., 2007. Conduction-based modeling of the biofilm anode of a microbial fuel cell. Biotechnol. Bioeng. 98, 1171-1182.

Marsili, E., Sun, J., Bond, D.R., 2010. Voltammetry and growth physiology of Geobacter sulfurreducens biofilms as a function of growth stage and imposed electrode potential. Electroanalysis 22, 865-874.

Mohd Yusoff, M.Z., Hu, A., Feng, C., Maeda, T., Shirai, Y., Hassan, M.A., Yu, C.-P., 2013. Influence of pretreated activated sludge for electricity generation in microbial fuel cell application. Bioresour. Technol. 145, 90-96.

Nercessian, O., Parot, S., Delia, M.-L., Bergel, A., Achouak, W., 2012. Harvesting electricity with Geobacter bremensis isolated from compost. PLoS ONE 7 , e34216.

Niessen, J., Schroder, U., Harnisch, F., Scholz, F., 2005. Gaining electricity from in situ oxidation of hydrogen produced by fermentative cellulose degradation. Lett. Appl. Microbiol. 41, 286-290.

Oh, S.-E., Yoon, J.Y., Gurung, A., Kim, D.-J., 2014. Evaluation of electricity generation from ultrasonic and heat/alkaline pretreatment of different sludge types using microbial fuel cells. Bioresour. Technol. 165, 21-26.

Pant, D., Van Bogaert, G., Diels, L., Vanbroekhoven, K., 2010. A review of the substrates used in microbial fuel cells (MFCs) for sustainable energy production. Bioresour. Technol. 101, 1533-1543.

Pocaznoi, D., Calmet, A., Etcheverry, L., Erable, B., Bergel, A., 2012a. Stainless steel is a promising electrode material for anodes of microbial fuel cells. Energy Environ. Sci. 5, 9645-9652.

Pocaznoi, D., Erable, B., Etcheverry, L., Delia, M.-L., Bergel, A., 2012b. Towards an engineering-oriented strategy for building microbial anodes for microbial fuel cells. Phys. Chem. Chem. Phys. 14, 13332-13343.

Rimboud, M., Desmond-Le Quemener, E., Erable, B., Bouchez, T., Bergel, A., 2015. The current provided by oxygen-reducing microbial cathodes is related to the composition of their bacterial community. Bioelectrochemistry 102, 42-49.

Rimboud, M., Pocaznoi, D., Erable, B., Bergel, A., 2014. Electroanalysis of microbial anodes for bioelectrochemical systems: basics, progress and perspectives. Phys. Chem. Chem. Phys. PCCP 16, 16349-16366.

Rivière, D., Desvignes, V., Pelletier, E., Chaussonnerie, S., Guermazi, S., Weissenbach, J., Li, T., Camacho, P., Sghir, A., 2009. Towards the definition of a core of microorganisms involved in anaerobic digestion of sludge. ISME J. 3 , $700-714$.

Torres, C.I., Krajmalnik-Brown, R., Parameswaran, P., Marcus, A.K., Wanger, G. Gorby, Y.A., Rittman, B.E., 2009. Selecting anode-respiring bacteria based on anode potential: phylogenetic, electrochemical, and microscopic characterization. Environ. Sci. Technol. 43, 9519-9524.

Tyagi, V.K., Lo, S.-L., 2013. Sludge: a waste or renewable source for energy and resources recovery? Renew. Sustain. Energy Rev. 25, 708-728.

Wang, Z., Mei, X., Ma, J., Wu, Z., 2012. Recent advances in microbial fuel cells integrated with sludge treatment. Chem. Eng. Technol. 35, 1733-1743.

Xiao, B., Yang, F., Liu, J., 2011. Enhancing simultaneous electricity production and reduction of sewage sludge in two-chamber MFC by aerobic sludge digestion and sludge pretreatments. J. Hazard. Mater. 189, 444-449.

Yang, F., Ren, L., Pu, Y., Logan, B.E., 2013. Electricity generation from fermented primary sludge using single-chamber air-cathode microbial fuel cells. Bioresour. Technol. 128, 784-787.

Yoshizawa, T., Miyahara, M., Kouzuma, A., Watanabe, K., 2014. Conversion of activated-sludge reactors to microbial fuel cells for wastewater treatment coupled to electricity generation. J. Biosci. Bioeng. 118, 533-539.

Zhi, W., Ge, Z., He, Z., Zhang, H., 2014. Methods for understanding microbial community structures and functions in microbial fuel cells: a review. Bioresour. Technol. 171, 461-468.

Zhu, X., Yates, M.D., Hatzell, M.C., Rao, H.A., Saikaly, P.E., Logan, B.E., 2014. Microbial community composition is unaffected by anode potential. Environ. Sci. Technol. $48,1352-1358$.

Zhu, X., Yates, M.D., Logan, B.E., 2012. Set potential regulation reveals additional oxidation peaks of Geobacter sulfurreducens anodic biofilms. Electrochem. Commun. 22, 116-119. 